\title{
Criminologie
}

\section{Le « rôle " de mères de détenus}

Une maternité confrontée aux contraintes carcérales et aux attaques sociales

\section{The role of mothers of prisoners}

\author{
Blame, constraints, and daily adaptation
}

El rol de las madres de los detenidos

Una maternidad confrontada a las restricciones carcelarias y a los ataques sociales

\section{Sandra Lehalle, et Mélissa Beaulieu}

Volume 52, numéro 1, printemps 2019

Les proches de personnes judiciarisées : expériences humaines et connaissances carcérales

URI : https://id.erudit.org/iderudit/1059543ar

DOI : https://doi.org/10.7202/1059543ar

Aller au sommaire du numéro

\section{Éditeur(s)}

Les Presses de l’Université de Montréal

ISSN

0316-0041 (imprimé)

1492-1367 (numérique)

Découvrir la revue

Citer cet article

Lehalle,, S. \& Beaulieu, M. (2019). Le « rôle » de mères de détenus : une maternité confrontée aux contraintes carcérales et aux attaques sociales. Criminologie, 52(1), 135-156. https://doi.org/10.7202/1059543ar

\section{Résumé de l'article}

Les recherches existantes sur les proches de personnes incarcérées abordent parfois le vécu des mères, mais elles s'intéressent très rarement à la spécificité du rôle maternel dans le contexte particulier de l'incarcération d'un enfant adulte. Sur la base d'une recherche empirique réalisée en 2017 au Canada, cet article propose une réflexion sur les représentations et les pratiques de la maternité envers un enfant adulte incarcéré. Nos résultats nous permettent d'explorer ce rôle nouveau auquel sont confrontées les mères qui découvrent et subissent les contraintes matérielles et institutionnelles que l'incarcération impose à leur maternité et comment elles s’y adaptent. Leurs expériences au sein et en dehors des murs de l'établissement carcéral se révèlent être marquées par des jugements sévères envers leur maternité passée et présente, qui postulent un lien de causalité entre la détention de leur enfant et leur déficit maternel. Nous relevons diverses stratégies pour faire face à ces nouvelles contraintes et à ce blâme qui leur est attribué par autrui et parfois par elles-mêmes. 


\title{
Le «rôle » de mères de détenus Une maternité confrontée aux contraintes carcérales et aux attaques sociales
}

\author{
Sandra Lehalle 1,2 \\ Professeure adjointe \\ Département de criminologie, Université d'Ottawa \\ slehalle@uottawa.ca \\ Mélissa Beaulieu \\ Candidate à la maîtrise \\ Département de criminologie, Université d'Ottawa \\ mbeau180@uottawa.ca
}

RÉSUMÉ - Les recherches existantes sur les proches de personnes incarcérées abordent parfois le vécu des mères, mais elles s'intéressent très rarement à la spécificité du rôle maternel dans le contexte particulier de l'incarcération d'un enfant adulte. Sur la base d'une recherche empirique réalisée en 2017 au Canada, cet article propose une réflexion sur les représentations et les pratiques de la maternité envers un enfant adulte incarcéré. Nos résultats nous permettent d'explorer ce rôle nouveau auquel sont confrontées les mères qui découvrent et subissent les contraintes matérielles et institutionnelles que l'incarcération impose à leur maternité et comment elles s'y adaptent. Leurs expériences au sein et en dehors des murs de l'établissement carcéral se révèlent être marquées par des jugements sévères envers leur maternité passée et présente, qui postulent un lien de causalité entre la détention de leur enfant et leur déficit maternel. Nous relevons diverses stratégies pour faire face à ces nouvelles contraintes et à ce blâme qui leur est attribué par autrui et parfois par elles-mêmes.

MOTS CLÉS - Détention, familles, mère, rôle, maternité, blâme.

1. Université d'Ottawa, Faculté des sciences sociales, 120, rue Université, Ottawa (Ontario), Canada, K1N 6N5.

2. Nous remercions chaleureusement les personnes qui ont accepté de participer à cette recherche, la Faculté des sciences sociales de l'Université d'Ottawa et le CRSH qui en ont financé la réalisation ainsi que Lisa Plamondon-Dufour pour ses relectures attentives.

Criminologie, vol. 52, $\mathrm{n}^{\circ} 1$ (2019) 


\section{Introduction}

Si la littérature scientifique a beaucoup analysé l'expérience carcérale du point de vue de l'établissement pénitentiaire ou de la personne incarcérée, les effets de l'incarcération sur les proches ont été, jusqu'au milieu des années 1990, largement ignorés des chercheurs. Certaines études se sont par la suite intéressées aux conséquences personnelles, conjugales, familiales et sociales de l'incarcération d'un proche, notamment aux États-Unis (Comfort, Grinstead, McCartney, Bourgois et Knight, 2005), au Canada (Hannem, 2008; Magnan, 2011 ; McCuaig, 2007) et en France (Cardon, 2002; Ricordeau, 2008; Touraut, 2012). Les recherches existantes ont également souligné l'ampleur des conséquences matérielles de l'incarcération sur les familles de détenus (Schwartz-Soicher, Geller et Garfinkel, 2011; Touraut, 2012). D'autres ont analysé l'impact psychologique et physique sur les proches (Hannem et Leonardi, 2014) et particulièrement les difficultés émotionnelles et comportementales observées chez les enfants (Dallaire, 2007; Hagan et Dinovitzer, 1999; Johnston et Sullivan, 2016).

Au gré de ces diverses thématiques, les recherches existantes abordent parfois le vécu des mères de détenus, mais s'intéressent très rarement à la spécificité du rôle de mère dans un tel contexte. Il semble pourtant pertinent de nourrir notre compréhension du vécu des proches de détenus des études sur la maternité et de la façon dont celle-ci est construite et interprétée socialement.

Les discours modernes sur la parentalité insistent sur le rôle de la socialisation, mais aussi de la prise en charge du bien-être émotionnel et du développement normatif des enfants (Beck-Gernsheim, 2002; Corrigan et Miller, 2004; Harden, 2005). La parentalité est orientée vers la production d'un enfant heureux, sain et bien socialisé. Dans une logique causale, les parents sont donc responsables du comportement de leur enfant (Backett-Milburn et Harden, 2004). Ils œuvrent à un projet de socialisation dont le succès est évalué dans la sphère privée par les parents eux-mêmes et dans la sphère publique par diverses institutions sociales (Harden, 2005). Pour Lapierre (2010), cette conception moderne de la parentalité engendre une augmentation de la responsabilité attribuée aux mères. En effet, la construction sociale dominante de la bonne mère impose à celle-ci de se conformer à certaines attentes: aimer, prendre soin et protéger ses enfants (Virokannas, 2011), être responsable (Miller, 2005), leur offrir un modèle positif et surtout faire passer leurs besoins en premier (Lapierre, 2007). 
Cette construction sociale hégémonique de la bonne mère fut analysée dès les années 1970 comme exerçant une forte pression sociale (Rich, 1976) sur les femmes et agissant comme un mécanisme normatif à travers lequel certaines manières de penser, de sentir et d'agir leur sont imposées (Goodwin et Huppatz, 2010). Spigel et Baraister (2009) argumentent que la plupart des femmes, en désirant être de bonnes mères, se conforment au scénario normatif prescrit par leur culture et s'autorégulent elles-mêmes. Pour Lapierre (2010), ce désir est à l'origine de stratégies dont l'objectif est de faire passer le soin et la protection de leurs enfants en premier.

Mais qu'en est-il lorsque l'enfant ne correspond pas à l'idéal normatif d'un enfant heureux, sain et bien socialisé?

Lorsque les enfants présentent des traits ou des comportements socialement indésirables, la littérature fait communément référence à un déficit parental (Etzioni, 1993), voire même à une construction sociale d'une identité de «mauvais parent» (Davis et Monago, 2016; Harden, 2005; Moses, 2010). La criminologie a souvent montré du doigt le déficit propre à la mère comme étant à l'origine du passage à l'acte délinquant.

Le rejet de la mère semble être plus criminogène que le rejet paternel. À partir de cette analyse, nous pouvons voir que les attitudes maternelles sont de première importance dans la genèse du crime. Si la mère était aimante, ses fils auraient eu moins de risques de devenir criminels - indépendamment du rejet paternel. (McCord et McCord, 1969, p. 108)

Diverses recherches soulignent en effet des divergences genrées dans les conceptions de la parentalité (Francis, 2012). Pour Davis et Manago (2016), la connexion présumée causale entre mère et enfant se fonde d'une part sur le discours scientifique sur les liens entre le bien-être de l'enfant et le corps de la mère responsable de la gestation et d'autre part sur le rôle traditionnellement attribué aux femmes dans le développement social de l'enfant. Badinter (1980) souligne le pas rapidement franchi entre responsabilité et culpabilité dès que l'enfant a le moindre problème et ne correspond pas à l'idéal social. La connexion causale avec l'enfant place le fardeau du blâme et de la honte sur la mère à laquelle on attribue une culpabilité morale (Davis et Manago, 2016).

Les recherches existantes sur les expériences de parents d'enfants atypiques (aux prises avec des problèmes de comportement ou de 
consommation, des problématiques de santé mentale ou des handicaps, etc.) révèlent des expériences de stigmatisation, de honte et de blâme devant leur échec à produire cet enfant idéal (Corrigan et Miller, 2004; Corrigan et al., 2006; Courcy et des Rivières, 2017; Moses, 2010).

Le blâme de la mère se manifeste dans l'environnement social (Courcy et des Rivières, 2017), mais il émane également de l'internalisation d'un propre sentiment de culpabilité de la mère en raison des attentes sociales et individuelles envers la maternité (Caplan, 1998). Les chercheurs qui ont étudié ce mécanisme d'autoblâme des parents ont relevé à la fois la souffrance et la détresse que cela engendre (Barrowclough, Tarrier, et Johnston, 1996; White et Barrowclough, 1998), mais également l'existence de mécanismes de gestion et d'adaptation (Hingley-Jones, 2005; Koro-Ljungberg et Bussing, 2009).

\section{Méthodologie}

Notre recherche vise à explorer les enjeux du rôle maternel dans le contexte particulier de l'incarcération d'un enfant devenu adulte. Comment une mère vit-elle cette incarcération et les bouleversements qui y sont associés? Comment gère-t-elle la situation et s'y adapte-t-elle?

Nous souhaitons donner la parole aux mères de personnes incarcérées pour mieux comprendre la diversité et la complexité de leurs expériences et situer ces expériences dans une compréhension plus large de la maternité, à travers ses composantes sociales et personnelles. Nous avons donc réalisé, en 2016-2017, une recherche empirique exploratoire au Québec et en Ontario auprès de seize proches, parmi lesquels neuf mères de personnes incarcérées. Les entretiens semi-directifs, d'une durée moyenne de 90 minutes, ont fait l'objet d'une analyse thématique avec le logiciel NVivo 11. Nous avons ainsi exploré les diverses manifestations de la peine dans la vie des proches, notamment les défis sociaux, économiques, relationnels et identitaires émanant des effets matériels et symboliques de l'incarcération d'un proche. Nous avons analysé comment ces personnes font face aux difficultés rencontrées et comment elles mobilisent certaines stratégies dans la gestion des dommages collatéraux de la sanction pénale.

C'est au fil de ces analyses thématiques qu'il nous a été donné de réfléchir à la situation spécifique des mères. Nous avons analysé leurs expériences en fonction de trois thématiques centrales, en l'occurrence 
les difficultés liées au milieu correctionnel, les réactions sociales auxquelles elles sont assujetties et le rôle de mère de famille. Cette dernière thématique a été approfondie selon quatre angles d'approche de leur rôle de mère: leurs perceptions, leurs aspirations, les attentes sociales et les changements constatés. Pour les analyser, nous avons mobilisé le concept d'identité de rôle développé par McCall et Simmons (1966) afin d'appréhender les perceptions et les attentes de soi qui sont rattachées au rôle présumé d'un individu.

Il convient de souligner que notre analyse des difficultés vécues par les mères n'a nullement pour objectif de les positionner comme des victimes passives mais bien de découvrir comment, faisant face aux nouvelles réalités et contraintes qui se présentent à elles, ces femmes sont amenées à faire des choix qui façonnent leur expérience de la maternité. De plus, nous sommes conscientes du biais de sélection évident de notre échantillon et notamment du fait que les expériences des mères qui ont accepté de participer à notre étude n'illustrent pas toutes les expressions de la maternité dans ce contexte bien particulier ${ }^{3}$. $\mathrm{Ne}$ cherchant pas à atteindre une représentativité quelconque, notre recherche exploratoire vise à développer une compréhension holistique de certaines représentations et pratiques de maternité envers l'enfant adulte incarcéré ${ }^{4}$.

Dans cet article, nous analyserons comment les mères dont les enfants ont des problèmes avec la justice vivent une expérience qui affecte inéluctablement la manière et la capacité qu'elles ont d'exercer leur rôle de mère. Nous évoquerons dans une première partie la découverte d'un nouveau rôle de mère de détenu qui prend conscience, subit et s'adapte aux contraintes de la détention. Nous aborderons dans une deuxième partie leur vécu des jugements relatifs à leur maternité ainsi que les stratégies qu'elles adoptent pour y faire face. Nous présenterons par la suite une réflexion sur les défis des représentations et des pratiques de la maternité en contexte d'incarcération d'un enfant adulte.

3. Il est même raisonnable de penser que des mères qui se seraient éloignées et désinvesties de leur enfant avant ou durant l'incarcération n'auraient pas répondu à notre appel à participants.

4. Nous avons ainsi volontairement exclu de l'analyse certaines variables telles que les caractéristiques sociodémographiques des participantes, la nature du crime commis par l'enfant adulte détenu, le type d'établissement correctionnel, etc. Si nous avons bien conscience que ces variables sont susceptibles d'influencer l'expérience des mères, nos résultats préliminaires laissent plutôt voir une constance des difficultés rencontrées lorsque l'analyse porte sur la maternité. 
Nous conclurons en évoquant brièvement les apports et les implications de notre recherche.

\section{Devenir une «mère de détenu »: un rôle nouveau, restreint et modelé par l'incarcération}

Apprendre qu'un de leurs enfants a commis une faute pour laquelle il sera puni par une peine d'emprisonnement est manifestement déconcertant et douloureux pour les mères. Elles doivent subitement assimiler cette nouvelle bouleversante et faire face aux réalités inattendues qui en découlent et qui modifient considérablement leur vie.

\section{La prise de conscience des réalités de l'incarcération et de ses conséquences}

Une fois passé le choc de l'incarcération, les mères vont rapidement ressentir une préoccupation constante quant au bien-être mental et émotionnel de la personne incarcérée lorsqu'elles réalisent l'étendue des privations imposées en détention.

Il fut un temps où je ne pouvais pas en parler sans pleurer, ça me choquait. Cela m'a consterné de penser que mon fils vivait cela; qu'il ait eu à endurer cela. Je ne sais pas comment l'on peut s'attendre à ce que les gens se comportent correctement lorsqu'ils n'ont rien à faire. C'est la recette pour un désastre, n'est-ce pas? Quand ils ne sont pas bien nourris. Quand ils ne peuvent pas avoir des douches appropriées et avoir une bonne hygiène. Quand ils doivent partager des coupe-ongles sales ${ }^{5}$. (Érika $\left.{ }^{6}\right)$

Elles découvrent la détention, mais réalisent en même temps l'ampleur de ce qu'elles ne savent pas. Leur préoccupation pour l'individu détenu est alors omniprésente: mange-t-il assez?, est-il en sécurité?, reçoit-il ses soins?, est-il suicidaire? Les mères rencontrées affirment avoir eu sérieusement peur pour la sécurité et l'intégrité physique de leur enfant.

Il y a aussi, comme, une terrible, une terrible peur. Une peur incroyable. [...] J'ai fait beaucoup de cauchemars dans lesquels il était mort et que je le regardais se faire tuer encore et encore. Parce que je n'ai vraiment aucune illusion: il n'est pas en sécurité. (Inès)

5. Dans cet article, nous avons traduit librement les citations anglaises.

6. Dans le but de préserver l'anonymat et la confidentialité des mères dont les propos seront cités à travers cet article, les noms de celles-ci ont été remplacés par des pseudonymes. 
Elles en savent assez pour être soucieuses, mais pas assez pour être rassurées. Au-delà de la peur du moment présent, ces craintes concernent également le futur et l'avenir, désormais incertain, de leur enfant. Les mères sont confrontées à une forme de deuil de la vie qu'elles souhaitent pour leur enfant détenu.

Je veux qu'il ait une vie. Et je continue à me dire qu'il aura une vie quand il sortira. Il semble que ce sera différent: le potentiel qu'il avait a beaucoup changé. [...] Il est vraiment brillant, mais ses chances d'obtenir un emploi quand il sortira sont minces, voire inexistantes. N'est-ce pas? Et je le regarde et il est beau et il était le meilleur papa que j'ai jamais vu et je doute qu'il ait d'autres enfants. (Inès)

L'incarcération est donc un moment de rupture avec le passé, le présent et le futur de leur enfant. Les mères qui ont participé à notre étude nous expliquent qu'elles vont alors anticiper les obstacles qui attendent leurs enfants à la remise en liberté. En conformité avec le rôle de la mère prévoyante et protectrice, elles mettent en place des stratégies pour faciliter l'avenir, telles que la création d'une entreprise de construction pour lui offrir un emploi à sa sortie, économiser et planifier l'héritage ou encore prévoir un hébergement.

Je ressens le besoin d'économiser, d'économiser, d'économiser, d'économiser. Que je travaille encore et que mon mari travaille encore, ainsi quand nous mourrons, ça ira! Nous allons laisser assez d'argent. (Érika)

\section{Découvrir les obstacles que l'incarcération impose à leur rôle de mère}

Les témoignages recueillis illustrent à quel point les implications matérielles de l'incarcération peuvent devenir des obstacles à la maternité. Les mères font face à une augmentation des charges à payer (les frais juridiques, les frais liés à l'éducation en détention, les dépôts d'argent au compte de la personne incarcérée, etc.). En effet, les coûts financiers et humains du maintien des liens mère-enfant sont souvent élevés. Elles mentionnent des factures téléphoniques mensuelles oscillant entre 350 et $800 \$$, auxquelles s'ajoutent les coûts liés aux visites dans des établissements trop souvent éloignés. Certaines entreprennent de déménager pour se rapprocher du lieu de détention. De façon très éloquente, une mère aborde notamment les lourds impacts financiers ayant résulté de son choix de s'investir, puis de demeurer présente et disponible auprès de son fils incarcéré: 
Nous avons déclaré faillite il y a quelques années... Ce n'est pas ce que j'avais planifié... Je pense que pour tout le monde, c'est une marche arrière financièrement... parce que ça coûte cher d'avoir un enfant en prison. (Kim)

La distance géographique entre le domicile familial et le lieu de détention engendre également des difficultés organisationnelles et pratiques qui sont des obstacles aux contacts mère-enfant.

Une fois, ma fille m'a appelée de Toronto. C'était juste incroyable. C'était un appel téléphonique incroyable. Je ne pouvais pas la visiter ici. (...) Je n'en avais pas les moyens. (Céline)

Aux défis matériels s'ajoutent les difficultés émanant du fonctionnement de l'établissement carcéral. Les procédures et techniques carcérales sont contraignantes et limitent souvent leurs pratiques de la maternité. Les mères sont fréquemment confrontées à des impératifs sécuritaires qui leur apparaissent disproportionnés et injustifiés. Pourquoi ne peuvent-elles pas envoyer des cartes postales coloriées à leur enfant incarcéré? Pour quelle raison, lors de visites familiales, un gardien fouille-t-il leurs sous-vêtements de rechange et impose-t-il l'utilisation de protections hygiéniques achetées préalablement par leur enfant à la cantine de l'établissement? Comment une mère atteinte d'un cancer peut-elle comprendre qu'elle soit obligée de retirer son foulard de chimio pour entrer? Et surtout, pourquoi les visites contacts ne sont-elles pas permises en détention préventive? Mal informées des procédures et non informées de leur raison d'être, les mères vivent beaucoup d'incertitude, de stress et d'épuisement qui se répercutent sur leur lien mère-enfant. Ne pas leur permettre de donner un baiser, un dessin... signifie en bout de compte que les procédures carcérales modèlent et redéfinissent leur façon d'être mère.

La capacité à fournir aide et soutien à l'enfant détenu se retrouve limitée par les conditions objectives de l'incarcération. Ces femmes sont désormais dans l'obligation de faire le deuil du rôle de mère qu'elles avaient et de celui qu'elles pensaient pouvoir avoir durant l'incarcération. À titre d'exemple, elles ne peuvent guère manifester leur soutien de la même manière qu'elles le faisaient autrefois ou de la façon dont elles désireraient le faire dans ces circonstances. Elles ne peuvent agir de façon spontanée et rendre visite à leur enfant quand elles le veulent ou le jugent nécessaire ni même le serrer dans leurs bras.

Ce qui est vraiment dur au début - et je l'ai souvent entendu répété par d'autres parents -, c'est que pendant quatre ans, je n'ai pas pu serrer mon fils dans mes bras. (Diane) 
Une femme rencontrée se demande même comment être mère si elle ne peut pas prendre son enfant dans ses bras dans ce moment difficile qu'il traverse.

\section{Agir en «bonne mère» en dépit des contraintes de l'incarcération}

Forcées de s'adapter aux réalités de l'incarcération qui limitent et réduisent leurs possibilités d'actions et d'interactions, les mères modifient et adaptent leur rôle aux formes qui sont dès lors permises. Elles vont exploiter au maximum les moyens de contact autorisés. Grâce au téléphone, certaines mères se rendent disponibles pratiquement en tout temps pour répondre aux besoins de leur enfant, pour les laisser ventiler, pour leur témoigner leur affection, pour leur donner de l'espoir, etc.

Vous savez, parfois il téléphone et a juste besoin de ventiler. Et devinez quoi? Je suis le sac de boxe. Et il va se fâcher et il sera frustré et il hurlerait et des choses comme ça... Mais l'appel téléphonique est leur seul lien vers l'extérieur et je sais que je suis la seule qui peut le calmer. (Kim)

Les mères deviennent le réceptacle des émotions de leur enfant et leur principal lien avec le monde extérieur. Les lettres sont utilisées très souvent pour maintenir le lien social de leur enfant au-delà des murs de l'établissement correctionnel.

Je lui envoie des photos pour qu'il puisse voir ce qui se passe. Je lui écris de temps en temps. [...] Il est assez déprimé en ce moment et j’ai fait des lettres composées d'images parce que la prison dans laquelle il est en ce moment, ils ne vous laissent avoir que trois photos. Donc, à la place, $j$ 'écris des lettres, je mets les photos dans le document Word et je l'imprime. Ça lui est autorisé. (Kim)

Le soutien offert peut se manifester sous d'autres formes: présence aux audiences, réalisation de démarches administratives, appui financier à l'éducation ou aux achats à la cantine, accompagnement des petits-enfants aux visites, etc. «Être là» pour son enfant détenu est principalement synonyme d'un investissement important en temps et énergie. Le quotidien des mères se retrouve rythmé par la gratification des besoins de l'enfant incarcéré auxquels elles souhaitent répondre à tout prix, au détriment parfois de leur bien-être. Le témoignage d'Inès, terrorisée par les procédures de contrôle des visites, illustre ce dévouement. 
«Le soutenir» [...] ce n'est pas facile. Les deux dernières nuits, je n'ai pas dormi... je me réveille littéralement quatre et cinq et six fois par nuit en rêvant de cela (les procédures de visites) parce que c'est si effrayant pour moi. Mais je vais quand même encaisser et y aller! Je prendrai la route et j'irai là-bas. (Inès)

En faisant constamment passer les besoins de leurs enfants avant les leurs, les mères adoptent les comportements prescrits et se conforment aux attentes véhiculées dans la construction sociale hégémonique de la bonne mère (Goodwin et Huppatz, 2010; Hays, 1996 ; Lapierre, 2007, 2010; Spigel et Baraister, 2009). Cette disponibilité pour l'enfant incarcéré a des implications bien concrètes dans la vie des mères, tant sur le plan personnel que professionnel.

Un mauvais jour pour mon fils, c'est six appels, c'est deux heures de ma journée. Les jours ne sont pas si longs. [...] Et les ambitions de ce que je pensais que je pourrais faire de ma vie; j'ai, en quelque sorte, mis cela en veilleuse. (Kim)

Certaines soulignent l'impossibilité d'avoir une carrière et de maintenir un emploi puisqu'elles souhaitent se rendre disponibles lors des procès, des visites et des appels. Pour Kim, la stratégie consistera à devenir travailleuse autonome:

Je n'aurais pas pu garder un emploi où je devais être là tout le temps parce que j'aurais dû trop m'absenter avec les crises de mon fils [...]. Je suis travailleuse indépendante, ce qui me permet d'avoir un peu de contrôle sur quand je fais quoi... (Kim)

L'incarcération d'un enfant bouleverse la vie et le rôle des mères. En contrôlant la qualité, la quantité et l'expression des relations avec l'enfant détenu, l'incarcération contraint et limite le rôle permis aux mères. Malgré ces contraintes et les efforts déployés pour répondre aux attentes sociales de la bonne mère qui fait passer son enfant en premier, elles sont confrontées aux diverses réactions sociales qui mettent à l'épreuve leur identité de rôle.

\section{Être une mère de détenus : confrontations aux jugements de sa maternité}

Une remise en cause avant tout personnelle

La première difficulté généralement rencontrée par une femme dont un enfant est en conflit avec la loi est la remise en question personnelle de la qualité de son rôle de mère. 
Vous vous blâmez. Je ne peux pas vous dire à quel point en tant que mère, vous vous blâmez. Vous regardez en arrière sur chaque expérience qu'ils ont eue comme enfants. "Comment ai-je géré cela? Étais-je trop dure? N'étais-je pas assez dure? Est-ce que je leur ai donné assez de temps et d'amour? Ai-je fait assez d'activités avec eux? À quel point les ai-je soutenus lorsqu'ils traversaient des moments difficiles?» Alors, en tant que mère, j'ai vraiment, vraiment douté de moi-même et je me suis blâmée. (Érika)

Le regard qu'elles portent sur elles-mêmes et l'évaluation qu'elles font de leur capacité à être les mères qu'elles aspirent à être sont profondément influencés par la construction sociale dominante de la «bonne mère» (Spigel et Baraister, 2009) ainsi que par les discours modernes sur la parentalité (Beck-Gernsheim, 2002; Corrigan et Miller, 2004; Harden, 2005). Ayant intériorisé l'idée selon laquelle elles devaient assurer la bonne socialisation de leurs enfants pour prévenir les conduites répréhensibles de leur part (Backett-Milburn et Harden, 2004), les mères vivent des sentiments d'inadéquation, de culpabilité ou de colère envers elles-mêmes, voire se sentent partiellement responsables du délit commis par leur enfant.

\section{Les réactions de la famille nucléaire bouleversée}

Si la famille nucléaire ne responsabilise pas les mères pour les événements ayant donné lieu à l'incarcération, elle réagit parfois à la façon dont celles-ci gèrent leur rôle de mère aussi bien auprès de la personne incarcérée qu'auprès des autres enfants. En consacrant leurs énergies au soutien de l'enfant incarcéré, elles sont parfois confrontées à des reproches quant au déséquilibre créé dans la cellule familiale. Les moments passés avec leurs autres enfants sont souvent interrompus par les appels de celui qui est incarcéré, appels auxquels elles se font un devoir de répondre en tout temps.

Cela a causé une dynamique étrange [...]. Surtout quand il appelle tous les jours et, quand il appelle, vous savez, cela dure vingt minutes. Donc, j'étais peut-être en train de faire quelque chose et tout à coup, c'est: «Oh! maman s'est encore absentée pour 20 minutes!» Ils ont du ressentiment vis-à-vis de cela $[. .$.$] . Tout le monde continue à manger. Je pars dans une autre pièce$ puis je reviens et je mange un repas froid. (Kim) 
En réponse à ce bouleversement, les enfants non incarcérés expriment des reproches et font comprendre aux mères que leur capacité à jouer adéquatement leur rôle s'est détériorée.

Elle m'a dit: «Maman, nous sommes toujours là. Regarde-nous. Nous sommes ici.» "Maman, nous sommes juste en face de toi.»Et donc, je devais faire une prise de conscience et me dire: qu'est-ce que je fais? En pensant que j'aide un problème et j'en crée un autre! (Fanny)

Certaines femmes interviewées disent avoir pris conscience de ce déséquilibre et affirment avoir ajusté leur rôle afin de «redevenir une bonne mère» pour tous.

\section{L'incertitude des réactions de l'entourage et des groupes sociaux fréquentés}

Le désir de protéger leur enfant et la peur d'être jugées comme mauvaises mères motivent parfois la décision de garder le secret au sein de la famille nucléaire.

Mais je n'en ai parlé à aucun de mes amis. À cause de cette perception... vous ne voulez pas que quelqu'un pense du mal de votre enfant. (Gina)

Lorsque les mères se confient à des proches, ceux-ci sont généralement enclins à leur offrir de l'aide pratique ou du soutien émotionnel pour les aider à faire face à cette épreuve. Ainsi, ils allègent partiellement le fardeau des mères et améliorent possiblement leur capacité à jouer leur rôle malgré la détention.

Ma sœur $\mathrm{C}^{\star \star \star}$ a été ma bouée de sauvetage. Je peux lui téléphoner n'importe quand. Quand je m'écroule, elle est là. Et $\mathrm{J}^{\star \star \star}$ (son mari): nous nous soutenons l'un et l'autre. (Inès)

Il arrive cependant qu'elles se sentent incomprises par certains de leurs proches qui contestent leur habileté à être de bonnes mères. Elles affirment faire l'objet de remarques et de conseils non sollicités qui remettent en cause leur capacité à prendre les bonnes décisions pour elles et tous les enfants de la famille.

J'ai un ami qui m'a dit: «Eh bien, tu devrais juste lui tourner le dos.» Quelqu'un m'a dit: «Jette l'éponge si tu veux minimiser tes pertes. Tu as deux autres enfants.» (Kim)

Dans les milieux sociaux qu'elles fréquentent au quotidien, milieu de travail, voisinage, communauté religieuse, etc., les femmes rencontrées 
affirment avoir peur d'être jugées. Pour cette raison, rares sont celles qui choisissent de volontairement divulguer l'incarcération de leur enfant. Lorsque le secret est, malgré elles, dévoilé, elles sont confrontées au jugement négatif et à des attitudes d'évitement et de distanciation.

Ils ont jugé, d'abord et avant tout. [...] Je travaillais avec beaucoup de personnel; peut-être quarante personnes. Et les gens qui avaient été très amicaux avant; ils ne me regardaient plus dans les couloirs! Ce fut une véritable prise de conscience! Comment j'ai été jugée... «Vous avez dû faire quelque chose. Que se passe-t-il dans votre famille?» J'étais traitée comme étant également coupable de l'infraction que mon fils avait commise. (Érika)

En réponse à ces expériences difficiles rattachées au secret à maintenir ou aux critiques à subir, certaines mères en concluront qu'il vaut mieux ne plus parler de leur enfant incarcéré. Ces femmes adoptent alors des stratégies d'évitement ou de rupture de liens. Corrigan et Miller (2004) ont relevé un phénomène similaire d'autoexclusion chez les membres de familles de personnes souffrant de troubles de santé mentale.

\section{La sévérité du jugement du personnel carcéral, du public et des médias}

Si l'ensemble des proches rencontrés dans le cadre de cette recherche témoigne d'une attitude trop souvent désobligeante de la part du personnel correctionnel, les mères affirment se sentir sévèrement jugées et traitées comme si elles étaient des criminelles elles-mêmes.

Vous avez l'impression d'être traitée comme si vous étiez une criminelle. Ils vous regardent avec des regards réprobateurs. Ils vous parlent rudement. [...] La majorité d'entre eux sont vraiment mal aimables. (Gina)

Leur rôle de mère est parfois directement remis en cause lors de ces interactions avec le personnel correctionnel.

L'expérience (visite dans une prison provinciale) était horrible. Horrible. Ils étaient grossiers... «Quel genre de mère?» Ce genre de chose. Quand j'ai nommé qui je venais visiter, c'était juste comme: «Hum. Ouais. Tu es la mère de ça?» (Fanny)

Des jugements similaires de leur capacité de mère émanent également du grand public. Les participantes déplorent le fait que les médias contribuent à alimenter une image négative d'elles par des jugements moraux portés sur leurs habiletés parentales. Les mères se sentent 
injustement montrées du doigt, critiquées, jugées, voire culpabilisées pour des actes dont elles ne sont guère les auteures.

Tout le monde dans la famille était jugé. "Woah, quel genre de famille est-ce? [...] Il doit y avoir quelque chose qui ne va pas avec leurs compétences parentales. Il doit y avoir quelque chose de mauvais dans leur famille, non? [...]» Donc, nous avons tous été jugés. (Érika)

Elles sont donc confrontées à la conception dominante de la responsabilité des mères en regard des comportements atypiques de leurs enfants (Backett-Milburn et Harden 2004; Cernkovich et Giordano, 1987).

\section{Un espace sécuritaire: les groupes de soutien}

Trop souvent incomprises, critiquées et jugées au sein des groupes sociaux qu'elles fréquentent, certaines mères de personnes incarcérées trouvent, auprès d'autres membres de familles de détenus, un espace où le blâme social de leur rôle de mère est absent. Comme l'indique l'une d'entre elles: «Tout le monde est assis autour de la table et nous avons tous le même squelette dans notre placard» (Érika).

Au sein d'un groupe de soutien constitué de mères de détenus, certaines affirment avoir trouvé les conditions propices au partage authentique de leurs émotions et de leurs expériences.

Ouais, c'est correct de pleurer dans le groupe. Nous sommes en sécurité. Nous pouvons nous laisser aller et exprimer nos émotions [...]. Il n'y a pas de jugement ici. Nous sommes toutes dans le même bateau. Nous n'avons pas à continuer à porter le faux masque de «je vais bien». Nous pouvons vivre notre souffrance et la gérer ensemble. (Fanny)

Il s'agit également d'une opportunité de donner un autre sens à leur expérience en aidant les autres mères.

Quand je croise d'autres femmes qui vivent la même chose, c'est ce que je leur dis en premier: «N'oubliez pas d'inclure vos autres enfants. Vous devez toujours les tenir au courant de ce qui se passe parce que, vous savez, cela peut devenir vraiment préjudiciable à la famille.» (Érika)

Nous observons toutefois que grâce à ces conseils sur la façon d'être une bonne mère dans cette situation, elles peuvent parfois reproduire et encourager entre elles la conformité aux attentes normatives de la bonne mère. 


\section{Discussion: les constructions personnelles et sociales du rôle de la bonne mère en contexte d'incarcération d'un enfant adulte}

\section{De «bonnes mères» dévouées malgré les obstacles}

L'incarcération d'un enfant adulte vient poser des limites et des contraintes au rôle de mère. Les procédures et les pratiques carcérales se révèlent être des obstacles importants à ce qui leur est possible de faire en tant que mères de personnes détenues. L'établissement carcéral, en s'immisçant dans la qualité et la quantité de l'expression du lien maternel, joue un rôle structurant sur leur façon d'être mères. Toutefois, en dépit du peu de contrôle sur la maternité qui leur est permise, les femmes rencontrées témoignent d'un sens particulièrement développé de responsabilité envers leurs enfants incarcérés. Nos résultats nous permettent, en effet, de contribuer à la littérature scientifique sur la maternité en déterminant comment, dans un contexte d'incarcération, les mères rencontrées jouent un rôle très actif de prise en charge de la socialisation, du bien-être émotionnel conforme aux discours modernes sur la parentalité (Beck-Gernsheim, 2002; Corrigan et Miller, 2004; Harden, 2005). Toutes les actions entreprises, les sacrifices faits et les stratégies mises en place ont bien pour objectif de contribuer au développement d'un enfant heureux, sain et bien socialisé. Elles agissent selon les critères de la construction sociale dominante de la bonne mère: tenter au mieux de prendre soin et de protéger son enfant (Virokannas, 2011) en faisant passer ses besoins en premier (Lapierre, 2007).

L'investissement maternel semble même être intensifié par l'incarcération de l'enfant adulte qui présente des besoins nouveaux, ou plutôt renouvelés, qui touchent aussi bien sa subsistance (argent pour la cantine, achats de vêtements, hébergement à la sortie, etc.) que sa socialisation. En effet, la plupart des stratégies mises en place visent le maintien du lien social de leur enfant. Notamment, les mères maximisent au mieux leurs ressources et les possibilités de contact avec la personne incarcérée au moyen du courrier et de visites. Pour certaines, le temps des visites devient un moment de tête-à-tête privilégié qu'elles n'avaient pas (ou plus) avant l'incarcération. Les mères font également preuve de ressources et de créativité pour que leur enfant incarcéré reste connecté avec sa famille et le monde extérieur par des gestes tels que les envois de magazines, de livres, de photos, de dessins, de matériel d'études. Au-delà de ce rôle de maintien immédiat du lien social, cer- 
taines stratégies ont pour objectif à moyen et à long terme d'assurer l'avenir de leur enfant après la détention. Qu'il s'agisse de déménager pour lui assurer un hébergement, de créer une entreprise indépendante pour lui assurer un emploi ou de ne pas prendre sa retraite et d'organiser son héritage pour lui assurer une sécurité financière, les mères anticipent les impacts négatifs persistants de l'incarcération et tentent de lui planifier un avenir.

Combler les besoins immédiats ou futurs de l'enfant détenu implique bien souvent des coûts personnels. En conformité avec le modèle de la mère idéale, les femmes rencontrées vont considérer les besoins de leur enfant incarcéré comme un impératif moral à prioriser, parfois aux dépens de leurs propres nécessités. Pour tenter de rendre la détention moins pénible et contribuer au bien-être de leur enfant incarcéré, les mères vont accepter de vivre ou de revivre des expériences qu'elles savent éprouvantes (liées à la détention ou aux écarts de conduite de leur enfant). En consacrant leur temps, leur argent et leur énergie à leur rôle de mère de la personne incarcérée, les mères font souvent passer au second plan leur carrière, leurs autres enfants, leur relation de couple, leur vie sociale, leurs économies et même leur qualité de vie. Des répercussions sur leur santé physique et mentale s'observent parfois lorsqu'elles évoquent angoisses, troubles du sommeil, dépression et augmentation de consommation (tabac, drogues, alcool, médicaments).

Nous avons bien conscience que cet investissement, ces stratégies et leurs conséquences ne sont pas exclusifs à la situation d'incarcération d'un enfant et peuvent être retrouvés chez les mères dans d'autres contextes. Cependant, le concept de soin donné à l'enfant prend un relief particulier dans notre étude puisque cet enfant est un adulte. L'incarcération engendre une situation non conforme à l'idée que lorsque l'enfant atteint l'âge adulte, le rôle parental de soin diminue. L'enfant adulte incarcéré présente soudainement un éventail de besoins particuliers très large dus à l'incarcération; ce qui rend possible pour les mères une extension du soin à prodiguer tout comme cela a été observé dans la littérature sur la maternité d'enfants atypiques.

Des mères jugées sur la base de la moindre éligibilité de leur enfant

Responsables d'être de bonnes mères qui comblent tous les besoins de leurs enfants (Lapierre, 2007, 2010), les mères sont donc les premières 
à se blâmer si ces derniers n'agissent pas de façon conforme. Selon le processus d'internalisation décrit par Caplan (1998), toutes les mères interviewées culpabilisent et se responsabilisent du sort de leur enfant. Nous pouvons dire que l'évaluation de réussite d'une bonne mère est en partie une autoévaluation réflexive basée sur les réussites de l'enfant (Beck et Beck-Gernsheim, 1995). Les femmes rencontrées luttent contre l'assimilation de la chute de leur enfant à leur échec en tant que mères durant les années de formation de leur enfant. Il semble bien que leurs expériences soient marquées par le discours dominant du modèle de mère déficiente qu'elles ont assimilé et qui est fréquemment insinué dans leurs rapports sociaux.

Toutes les mères interviewées dans le cadre de cette recherche font en effet face au jugement négatif du comportement de leur enfant pour lequel elles sont responsabilisées. En dépit d'exceptions positives, le blâme social relevé par Courcy et des Rivières (2017) émane des groupes qu'elles fréquentent, des employés des établissements correctionnels, mais également de leur entourage proche. Il prend la forme de commentaires et de réflexions qui remettent en cause leur rôle de mère passé et présent. Nous analysons ces manifestations du blâme social comme des attaques à la maternité (Heward-Belle, 2017), c'està-dire des techniques de contrôle et de régulation des expériences, des identités et des pratiques de maternité. Ces attaques prennent place dans un contexte où des constructions genrées et hégémoniques dictent ce que signifie être une bonne mère dans une société et à une époque données (Goodwin et Huppatz, 2010, p. 1-2). Il s'agit bien d'une forme de contrôle coercitif spécifique aux femmes et à leur expérience de devenir et d'être mère (Heward-Belle, 2017).

La réaction sociale à laquelle elles sont confrontées semble impliquer qu'elles ont échoué à être une bonne mère à la fois durant les années de formation de leur enfant et dans leur gestion de l'incarcération de celui-ci à l'âge adulte. Les femmes rencontrées témoignent en effet de critiques ou de reproches plus ou moins subtils de leurs pratiques de la maternité dans le cadre de l'incarcération. Accorder du temps et donner de l'argent, pourtant des comportements conformes au modèle de la bonne mère, deviennent parfois des pratiques réprouvées quand l'enfant est incarcéré. À la différence des enfants atypiques dont les difficultés sont appréhendées comme étant innées ou tout au moins involontaires (handicaps, maladies, troubles de santé mentale), les actes ayant conduit à l'incarcération sont socialement interprétés comme des choix faits par 
l'enfant adulte qui perdrait ainsi sa légitimité à bénéficier du dévouement de sa «bonne mère». La réaction sociale vécue par les mères semblerait en effet suggérer une inversion du modèle de la bonne mère fondé sur la doctrine de moindre éligibilité qui prescrit pour les contrevenants aux lois des conditions de vie nécessairement moins favorables que celles des citoyens libres les plus pauvres (Kaminski, 2010).

La moindre éligibilité ne disparaît pas aussi nettement qu'ailleurs dans le champ pénitentiaire, tant le comportement coupable du délinquant (...) légitime la discrimination et tant persiste la représentation religieuse d'une punition qui doit représenter l'enfer sur terre. (Kaminski, 2010, p. 214)

Selon une telle perspective, la personne incarcérée serait moins méritante que le citoyen respectueux de la loi, ce qui justifierait à son égard un moindre respect et un traitement différentiel. Notre analyse avance que la doctrine de moindre éligibilité serait élargie et dépasserait les conditions d'incarcération pour atteindre également les soins prodigués par les mères à leur enfant incarcéré. En effet, cette doctrine est susceptible, aux yeux de certains, de modifier, voire d'inverser, les attentes sociétales en ce qui a trait à la maternité durant l'incarcération d'un enfant adulte. Non seulement l'incarcération pose des entraves aux exigences et aux pressions véhiculées dans la construction sociale de la «bonne mère», mais celles-ci en sont même découragées. Elles se voient dès lors critiquées, jugées et blâmées pour avoir fait le choix de soutenir l'enfant incarcéré en dépit du crime pour lequel il est incarcéré.

\section{Conclusion}

En exposant les répercussions de l'incarcération sur la maternité des mères d'enfants adultes incarcérés, notre recherche contribue de façon évidente à la littérature croissante sur les diverses conséquences collatérales de notre système pénal. Mais de façon plus originale, nos résultats amorcent un dialogue jusque-là délaissé entre la littérature criminologique et la littérature sur la parentalité et plus spécifiquement la maternité. Cette étude montre, en effet, comment les mères rencontrées expriment par leurs actions une volonté persistante de se conformer à la construction sociale dominante du rôle de la bonne mère en dépit des contraintes particulières imposées par l'incarcération. Devant un environnement adverse qui met durement à l'épreuve leur maternité, leur investissement, intensifié au moyen de diverses stratégies, vient en fait remettre en cause le modèle de déficit parental souvent privilégié 
dans l'analyse de la délinquance. Malgré cela, les mères d'enfants adultes incarcérés sont confrontées à diverses manifestations du blâme social quant à leur maternité passée et présente; blâme qu'elles peinent à ne pas s'attribuer elles-mêmes. Ces attaques (Heward-Belle, 2017) à leur maternité visent à contrôler et à réguler les expériences, les identités et les pratiques de la maternité dans le contexte de l'intervention pénale.

Nos résultats invitent, sur divers plans, à rompre avec les préjugés persistants quant aux «mauvaises mères d'adultes délinquants». Globalement, nous préconisons de mobiliser dans les médias un contrediscours aux opinions préconçues du public sur les mères d'adultes incarcérés. De plus, nous recommandons d'offrir des formations appropriées au personnel correctionnel qui travaille auprès des familles. Au-delà d'un discours trop souvent vide de contenu sur l'importance des liens familiaux dans la réinsertion sociale des personnes judiciarisées, il convient concrètement de briser sur divers fronts les obstacles inutiles à la maternité en respectant les choix de celles qui entreprennent de soutenir leur enfant adulte incarcéré.

\section{Références}

Backett-Milburn, K. et Harden, J. (2004). How children and their families construct and negotiate risk, safety, and danger. Childhood, 11(4), 429-447.

Badinter, E. (1980). L'amour en plus. Histoire de l'amour maternel. Paris, France: Flammarion.

Barrowclough, C., Tarrier, N. et Johnston, M. (1996). Distress, expressed emotion, and attributions in relatives of schizophrenia patients. Schizophrenia Bulletin, 22(4), 691-702.

Beck, U. et Beck-Gernsheim, E. (1995). The normal chaos of love. Cambridge, Royaume-Uni: Polity Press.

Beck-Gernsheim, E. (2002). We want a special child. Dans E. Beck-Gernsheim (dir.), Reinventing the family: In search of new lifestyles (p. 86-104). Cambridge, Royaume-Uni : Polity Press.

Caplan, P. J. (1998). 'Mother-blaming'. Dans M. Ladd-Taylor et L. Umansky (dir.), 'Bad' mothers: The politics of blame in twentieth-century America (p. 127144). New York, NY: New York University Press.

Cardon, C. (2002). Relations conjugales en situation carcérale. Ethnologie française, 32(1), 81-88.

Cernkovich, S. A. et Giordano, P. C. (1987). Family relationships and delinquency. Criminology, 25(2), 295-319.

Comfort, M., Grinstead, O., Mccartney, K., Bourgois, P. et Knight, K. (2005). "You can't do nothing in this damn place": Sex and intimacy among couples with an incarcerated male partner. The Journal of Sex Research, 42(1), 3-12. 
Corrigan, P. W. et Miller, F. E. (2004). Shame, blame, and contamination: A review of the impact of mental illness stigma on family members. Journal of Mental Health, 13(6), 537-548.

Corrigan, P. W., Watson, A. C. et Miller, F. E. (2006). Blame, shame, and contamination: The impact of mental illness and drug dependence stigma on family members. Journal of Family Psychology, 20(2), 239-246.

Courcy, I. et des Rivières, C. (2017). "From cause to cure": A qualitative study on contemporary forms of mother blaming experienced by mothers of young children with autism spectrum disorder. Journal of Family Social Work, $20(3), 233-250$.

Dallaire, D. H. (2007). Incarcerated mothers and fathers: A comparison of risks for children and families. Family Relations, 56(5), 440-453.

Davis, J. L. et Manago, B. (2016). Motherhood and associative moral stigma: The moral double bind. Stigma and Health, 1(2), 72-86.

Etzioni, A. (1993). Parenting deficit. Londres, Royaume-Uni: Demos.

Francis, A. (2012). Stigma in an era of medicalization and anxious parenting: How proximity and culpability shape middle-class parents' experiences of disgrace. Sociology of Health or Illness, 34(6), 927-942.

Goodwin, S. et Huppatz, K. (2010). The good mother: Contemporary motherhoods in Australia. Sydney, Australie: Sydney University Press.

Hagan, J. et Dinovitzer, B. (1999). Collateral consequences of imprisonment for children, communities and prisoners. Crime and Justice, 1(26), 121-162.

Hannem, S. N. (2008). Marked by association: Stigma, marginalisation, gender and the families of male prisoners in Canada (Thèse de doctorat, Université Carleton). https://doi.org/10.22215/etd/2009-06678

Hannem, S. N. et Leonardi, L. (2014). Family victim research: Needs and characteristics Ontario region - Final report. Regroupement canadien d'aide aux familles des détenu(e)s.

Harden, J. (2005). Parenting a young person with mental health problems: Temporal disruption and reconstruction. Sociology of Health and Illness, 27(3), 351-371.

Hays, S. (1996). The Cultural Contradictions of Motherhood. New Haven: Yale University Press.

Heward-Belle, S. (2017). Exploiting the 'good mother' as a tactic of coercive control. Affilia, 32(3), 374-389.

Hingley-Jones, H. (2005). An exploration of the issues raised by living with a child with autistic spectrum disorder and a professional's attempt to move beyond pity and blame. Journal of Social Work Practice, 19(2), 115-129.

Johnston, D. et Sullivan, M. (2015). Parental incarceration personal accounts and developmental impact. New York, NY: Routledge.

Jones, D. W. (2004). Families and serious mental illness: Working with loss and ambivalence. British Journal of Social Work, 34(7), 961-979.

Kaminski, D. (2010). Droits des détenus, normalisation et moindre éligibilité. Criminologie, 43(1), 199-226. 
Koro-Ljungberg, M. et Bussing, R. (2009). The management of courtesy stigma in the lives of families with teenagers with ADHD. Journal of Family Issues, 30(9), 1175-1200.

Lapierre, S. (2007). Taking the blame?: Women's experiences of mothering in the context of domestic violence (Thèse de doctorat, University of Warwick). Repéré à https://search.proquest.com/docview/899696292/

Lapierre, S. (2010). Striving to be 'good' mothers: Abused women's experiences of mothering. Child Abuse Review, 19(5), 342-357.

Magnan, I. (2011). Être parent d'un fils adulte détenu: conséquences et soutien social (Mémoire de maîtrise, Université du Québec à Chicoutimi). Repéré à http:// constellation.uqac.ca/2404/1/030288541.pdf

McCall, G. J. et Simmons, J. L. (1966). Identities and interactions. New York, NY: Free Press.

McCord, W. M. et McCord, J. (1969). Origins of crime: A new evaluation of the Cambridge-Somerville Youth Study. Montclair, NJ: Patterson Smith.

McCuaig, E. (2008). Doing time on the outside Managing relationships with imprisoned men (Mémoire de maîtrise, Université d'Ottawa). Repéré à http://hdl. handle.net/10393/28006

Miller, T. (2005). Making sense of motherhood: A narrative approach. Cambridge, Royaume-Uni: Cambridge University Press.

Moses, T. (2010). Exploring parents' self-blame in relation to adolescents' mental disorders. Family Relations, 59(2), 103-120.

Rich, A. (1976). Ofwoman born. Motherbood as experience and institution. Londres, Royaume-Uni: Virage Press.

Ricordeau, G. (2008). Les détenus et leurs proches. Solidarités et sentiments à l'ombre des murs. Paris, France: Autrement.

Schwartz-Soicher, O., Geller, A. et Garfinkel, I. (2011). The effect of paternal incarceration on material hardship. Social Service Review, 85(3), 447-473.

Spigel, S. et Baraister, L. (2009). Editorial. Studies in the Maternal, 1(2), 1-3. http://doi.org/10.16995/sim.103

Touraut, C. (2012). La famille à l'épreuve de la prison. Paris, France: Presses universitaires de France.

Virokannas, E. (2011). Identity categorization of motherhood in the context of drug abuse and child welfare services. Qualitative Social Work, 10(3), 329-345.

White, C. et Barrowclough, C. (1998). Depressed and non-depressed mothers with problematic preschoolers: Attributions for child behaviours. British Journal of Clinical Psychology, 37(4), 385-398.

\section{The role of mothers of prisoners: blame, constraints, and daily adaptation}

ABSTRACT - Existing research on the relatives of prisoners sometimes addresses the experiences of mothers, but rarely focuses specifically on the maternal role in the context of the incarceration of a child who has reached adulthood. This article, based 
on empirical research conducted in 2017 in Canada, explores the representations and practices of maternity towards an incarcerated adult child as new roles are explored and they discover, undergo, and adapt to the material and institutional constraints imposed by the incarceration of their adult child. Their experiences within and outside prison walls are marked by harsh judgments about their past and present mothering that often postulate a causal link between their child's detention and a deficit in their mothering. We identify various strategies that can be used to cope with both these new constraints and the blame attributed to them by others and sometimes by themselves.

KEYWORDS - Incarceration, family, mother, motherhood, blame.

\section{El rol de las madres de los detenidos: una maternidad confrontada a las restricciones carcelarias y a los ataques sociales}

RESUMEN - Las investigaciones existentes sobre los familiares de las personas encarceladas a veces abordan las experiencias de las madres, pero estas se interesan muy poco por la especificidad del rol maternal en el contexto particular del encarcelamiento de un hijo adulto. Basándonos en una investigación empírica realizada en Canadá en el 2017, este artículo propone una reflexión sobre las representaciones y las prácticas de la maternidad hacia un hijo adulto encarcelado. Los resultados nos permiten explorar este nuevo rol al cual están confrontadas las madres que descubren y padecen las restricciones materiales e institucionales que el encarcelamiento le impone a la maternidad, y cómo ellas se adaptan. Se revela que sus experiencias en el seno y por fuera de los muros de la institución carcelaria están marcadas por juicios severos hacia su maternidad pasada y presente que postulan una relación causal entre la detención de su hijo y su déficit maternal. Relevamos diversas estrategias para enfrentar estas nuevas restricciones y esta culpabilidad que les es atribuida por los otros, y a veces por sí mismas.

PALABRAS CLAVE - Detención, familias, madre, rol, maternidad, culpa. 